\title{
A REVIEW OF ACOUSTIC SPACE FILLING CURVE METAMATERIALS FOR JET ENGINE INLETS
}

\author{
JN Glover PhD Student, Department of Aeronautical and Automotive Engineering, \\ Loughborough University, UK \\ DJ O'Boy Senior Lecturer in Structural Dynamics, Department of Aeronautical and Automotive \\ Engineering, Loughborough University, UK
}

\section{INTRODUCTION}

Aircraft are notorious for their large sound profile, yet noise is one of the most significant limiting factors for aviation growth. In 2011 the World Health Organisation identified environmental noise as the second largest environmental health risk in Western Europe [1]. Prolonged exposure to unacceptable noise levels ( $\geq 55 \mathrm{~dB}$ Lden (day evening-night level)) is linked to conditions including tinnitus, hypertension, coronary heart disease and myocardial infarctions [2]. Factor such as these have led organisations like the Advisory Council for Aircraft Research and Innovation in Europe (ACARE) to target a reduction in perceived noise by $50 \%$ for new aircraft entering into service in 2020 relative to the year-2000 [3]. Moreover, airport operations are often restricted at night, due to factors such as noise therefore noise control offers a potential increased capacity.

The ambitious ACARE targets are not being met, as of 2018 the percentage reduction is at $32 \%$ and is only projected to reach $37.5 \%$ [4]. The dominant noise source on an aircraft is the engine; approximately $68 \%$ at approach and at take-off approximately $98 \%$ [5]; however current technology and improvement attempts are not having the desired impact. This research project aims to introduce this innovation with metamaterial acoustic liners for the jet engine inlet. Metamaterials are a class of material that are engineered to produce properties that do not naturally occur. In practical terms this means additional properties of attenuation can be added to traditionally used aerospace materials by changing the internal structure. Metamaterials are not governed by the traditional bulk modulus and density characteristics of materials thus capable of controlling acoustic patterns through zero or even negative refraction; making acoustic cloaking possible and subwavelength low frequency attenuation [6]. In particular Space Filling Curves (SFC) have the potential to provide a lightweight, thin, high performance acoustic liner [7].

This paper contains a comparison of some of the most promising metamaterial acoustic liner designs which utilise Space Filling Curves, in terms of the fundamental theory of the design category and a discussion of the reflection and absorption characteristics. Computer simulation and impedance tube based experimental testing compares and contrasts the designs and the paper concludes with future application for aeroacoustics with particular focus on the engine inlet.

\section{ACOUSTIC LINERS}

Typical acoustic liners for a commercial jet engines use the principle of a Helmholtz Resonator (HR). For many decades panels with multiple HRs have been the standard acoustic liner being cheap, relatively lightweight and effective for one dominant frequency. However, as aviation moves into a more environmentally conscious era innovations are needed. The HR suffers from a narrowband attenuation and poor low frequency attenuation due to the mass law. The effect of the mass law is essentially, the lower the desired attenuation frequency the larger the liner depth needs to be [8] (and for the purposes of this paper the frequency range of interest is $200-2000 \mathrm{~Hz}$ ).

Vol. 42. Pt. 1. 2020 
The crucial focus for jet engine applications are the dominant frequencies which are low frequency, with the lowest at approximately $630 \mathrm{~Hz}$ [9]. Incidentally, if the $630 \mathrm{~Hz}$ limit was required to be attenuated by a typical liner, the depth would need to be increased up to 10 inches [9]. Moreover, the industrial trend towards ultra high bypass engines (based on the desire to reduce engine emissions) looks to increase the bypass ratio from 8 to 15 [10]. The consequence is an increase in weight and potential drag which is predicted to be countered by a reduction in the thickness of the outer casing, meaning that any soundproofing contained within needs to become thinner [10].

\subsection{Helmholtz resonator}

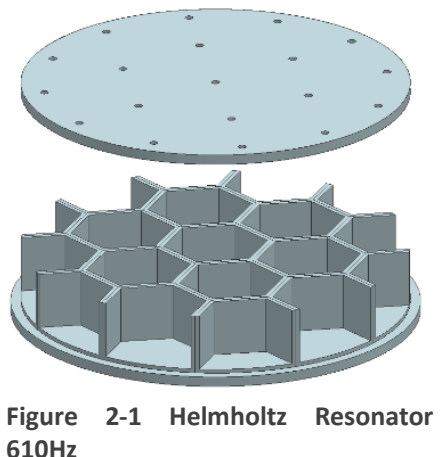

Traditional Helmholtz Resonators (HR) are used to control steady, simple harmonic sound at a narrow frequency range. The main advantages of this resonator is its simplicity, although careful tuning is required for effective noise attenuation [8]. The HR works as an acoustic stopband filter with the action of the volume of air in the cavity emulating a mass spring system [8]. However, this causes an undesirable back pressure which has a detrimental effect on the efficiency and performance of the engine. The limited narrow band can be improved with a range of tuned HRs, but this has a proportional increase in back pressure [9]. If frequency bands could be filtered out through an alternative method such as multi-resonant scattering, there would be a far smaller effect on engine efficiency which could be a designed in characteristics of metamaterial liners.

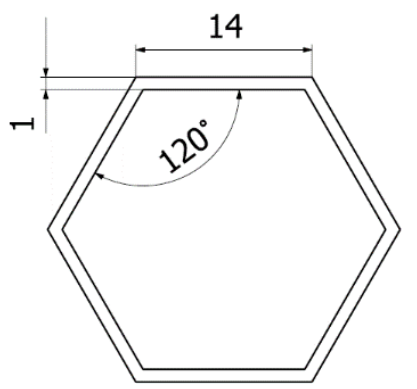

Figure 2-2 Helmholtz Resonator $610 \mathrm{~Hz}$ unit cell
For the purposes of comparison in this paper a baseline acoustic liner was designed using a hexagonal $610 \mathrm{~Hz}$ tuned HR (Figure 2-1 and Figure 2-2), see formula in [10]. This was chosen to compromise between industry standard design, the working frequency range of the experimental impedance tube and available 3D printing capabilities. The hexagonal HR liner was a benchmark of success criteria for the SFC liners. Thus, a good liner would show a better absorption coefficient at a wider frequency range and ideally at a lower weight. The additional characteristics of a typical sandwich configuration was also applied to the SFCs for the following three reasons: 1) The two microphone impedance set up doesn't allow for the grazing configuration in which metamaterials used being traditional designed to record head-on resonance for sandwich

liners. 2) A sandwich configuration is the current standard for soundproofing thus can be considered commercial. 3) The additional resonant conditions are predicted to improve absorption coefficients for the SFC samples.

\subsection{Space Filling Curves}

A space-filling curve (SFC) metamaterials are designed with a sub-wavelength curled wave path creating a mazelike pattern (Figure 2-3). SFC were developed from a purely mathematical problem where a line passes through every cell element of a grid, so that every cell is visited exactly once. The primary physical mechanism of noise reduction is an extended pathlength compared to unit depth which enables subwavelength attenuation. Different designs can offer multiple propagation paths, directions and scattering. Wang et al [11] has proposed an equivalent model for SFC which applies HR and quarter wavelength tube theory to a ranging transfer matrix that defines the SFC peak frequency. The transfer matrix establishes a relationship between the acoustic pressure and volume velocities in 2 defined regions. 
Equation 2-1- Equivalent model transfer matrix

$$
\left[\begin{array}{l}
p_{\text {in } I} I \\
U_{\text {in_I }_{I} I}
\end{array}\right]=T_{1} T_{2}\left[\begin{array}{l}
p_{\text {out_II }} \\
U_{\text {out }_{-} I}
\end{array}\right]=\left[\begin{array}{ll}
A & B \\
C & D
\end{array}\right]\left[\begin{array}{l}
p_{\text {out } I I I} \\
U_{\text {out_II }}
\end{array}\right]
$$

Equation 2-1 shows the two regions the first is a straight region which is the straightened coiling path equated to a resonator neck and the second is a cavity region which would be designed voids. When region 1 tends to zero HR theory alone applies and when region 2 tends to zero the quarter wavelength theory alone applies.

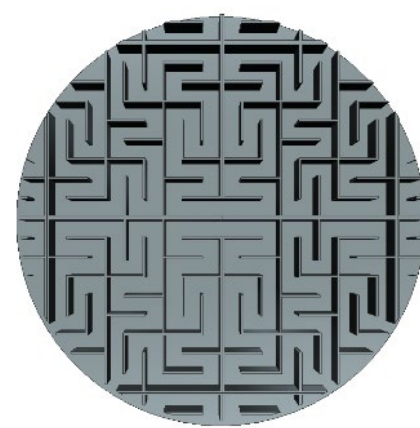

Figure 2-3 Wunderlich space filling curve

In this paper three designs are being compared termed hexagonal, labyrinth and zig-zag. These designs are tested in a closed 2 microphone impedance tube with a head on flow which differs from the origin papers testing method of grazing flows. The 2 microphone impedance tube is an industry standard test used to establish the acoustic characteristics of each liner in standardised conditions. SFC's have the advantage of being ultra-thin and lightweight, having comparable attenuation to traditional liners at a fraction of the size (a claim which was challenged as part of this paper). They can also achieve broadband attenuation with multiple units unlike Helmholtz resonators and due to advances in 3D printing they are easy to manufacture. Conversely, as these are fairly recent advancements, there are limited examples of commercial use and limited experimental verification with multiple studies simulation only.

\subsubsection{Symmetry broken hexagonal metamaterial}

The first acoustic liner design investigated was proposed by Xia et al. and termed a "Symmetrybroken metamaterial". The design was iterated by extending the one-dimensional chain to a twodimensional lattice, a bi-periodic two-dimensional metamaterial can be realised by a so-called hexagonal lattice [12]. The sample presented by Xia et al. has tessellated hexagonal lattices with a central void tested using simulation in $2 \mathrm{D}$ on COMSOL only. The design creates a subwavelength monopole resonance $367 \mathrm{~Hz}$ from in-phase propagation and dipole resonance $753 \mathrm{~Hz}$ through multiple scattering.

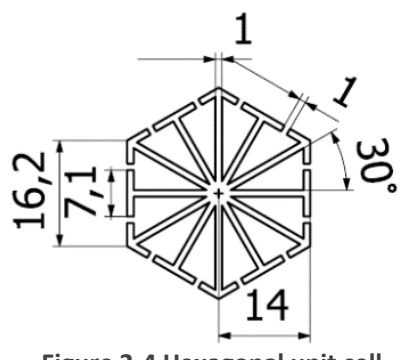

Figure 2-4 Hexagonal unit cell

The hexagonal liner sample design Figure 2-4 and sizing was inspired by Xia et al. "Symmetry-broken metamaterial". It was progressed into $3 \mathrm{D}$ with a nominal depth matching that of the baseline HR. This test liner like all the designs investigated was tessellated (See Figure 2-3 the tessellation of Figure 2-6.) to cover the whole $95 \mathrm{~mm}$ diameter space available in the test rig (See Figure 3-1). No design had voids for consistency, although some origin papers such as this did. Xia et al. recorded zero transmission at monopole and dipole and as the number of units present in the flow increases the overall average absorption increases. The double resonance and low frequency range are both aligned to the dominate frequencies of a jet engine inlet applications.

\subsubsection{Spiral Labyrinth metamaterial}

The "Spiral Labyrinthine acoustic metamaterial" or SLAM developed by Liu et al. is the second design under investigation in this paper [13]. This design offers an elongated transmission path of sound propagation much larger than the external dimensions offering sub-wavelength attenuation. It 
is mathematically based on scaled triangles descending of ratios $1 / 16,1 / 8,1 / 4$, and $1 / 1$ and tested using simulation in 2D on COMSOL only. Unlike the Hexagonal or Zig-Zag designs, the "spirallabyrinthine" allows multiple direction propagation increasing the impedance as energy is converted to heat through wall friction or from opposing flow orientation.

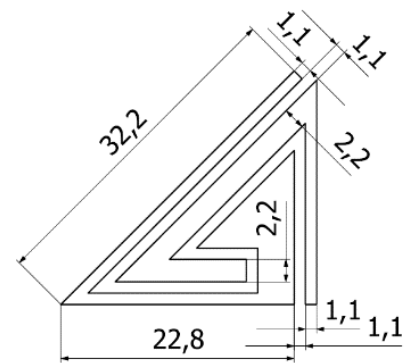

Figure 2-5 Labyrinthine unit cell

This design termed labyrinthine (Figure 2-5) was chosen due to its extremely high filling ratio being in direct contrast to the zig-zag detailed below. Filling ratio being the percentage wall volume to wave path volume. In addition, the ratio of the scaled triangles should allow for high level of tailoring and therefore frequencies of interest. A labyrinthine liner sample design and sizing was developed based upon the work by Lui et al., again it was developed at the same depth as the HR sample to produce a 3D design. This design offered significant absorption $>99 \%$ for $100 \mathrm{~dB}$ at $1158 \mathrm{~Hz}$ and $91 \mathrm{~dB}$ at $2122 \mathrm{~Hz}$. Although only one of the peak values is within the $200-2000 \mathrm{~Hz}$ working frequency range the high absorption is very promising for aeronautical applications. Similarly to the symmetry broken hexagonal metamaterial [12] research, as the number of units present in the flow increases, the overall absorption increases.

\subsubsection{Zig-Zag metamaterial}

The "Labyrinth liner" developed by Krushynska et al. [14] is based upon a Wunderlich space-filling curve where the path ratio is $3^{N}-1 / 3^{N}$. The "Labyrinth liner" utilises the path elongation characteristics changing the wave speed through the path when compared to background medium. For instance, the width of the unit is $41 \mathrm{~mm}$, but the path length is approximately $393 \mathrm{~mm}$ therefore time to propagate through the unit is vastly increased when compared to a simple cavity such as a $\mathrm{HR}$. There is also additional impedance from wall friction due to the large wall length thus it is a subwavelength attenuator. The research by Krushynska et al. covered an extensive frequency range between $60 \mathrm{~Hz}$ and $9 \mathrm{kHz}$ encompassing the full range of a jet engine noise profile. This research (unlike the hexagonal and labyrinth) built on the experimental validation of Cheng et al. [15] and Xie et al. [16], to investigate tortuosity and path dimensions in 3D. Concluding that increasing the tortuosity and elongating a wave path, can achieve almost perfect reflection which although prevents sound propagation can increase back pressure within the engine reducing performance and efficiency.

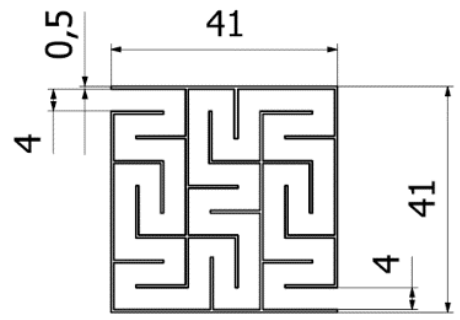

Figure 2-6 Zig-Zag unit cell
This design was chosen due to its low filling ratio; the simplicity of design and the potential ease of unit scaling. The second unit iteration was recreated termed zig-zag which showed absorption peaks at $500-4000 \mathrm{~Hz}$ at $\sim 500 \mathrm{~Hz}$ steps ideally in the $200-2000 \mathrm{~Hz}$ range of interest [14]. The depth was used as defined in the origin paper $15 \mathrm{~mm}$ unlike the other samples at $20 \mathrm{~mm}$. The second iteration (Figure 2-6) showed an extremely high refractive index whilst maintaining a low overall weight and compact design. The zig-zag design was sketched in the $x$-y plane although future iterations will investigate the $x-z$ plane.

\section{IMPEDANCE TUBE RIG}

\subsection{Impedance tube theory}

A two microphone Impedance Tube (Figure 3-1) was designed and built to accurately measure sound reflection and absorption coefficients according to ISO10534-2 [17]. The basic principle of the tube is that a plane wave is generated by a sound source and measurements of acoustic pressure are taken at fixed locations depending on the number of microphones. The reflection or

Vol. 42. Pt. 1. 2020 
absorption through the sample generates a standing wave that can be measured by the microphones.

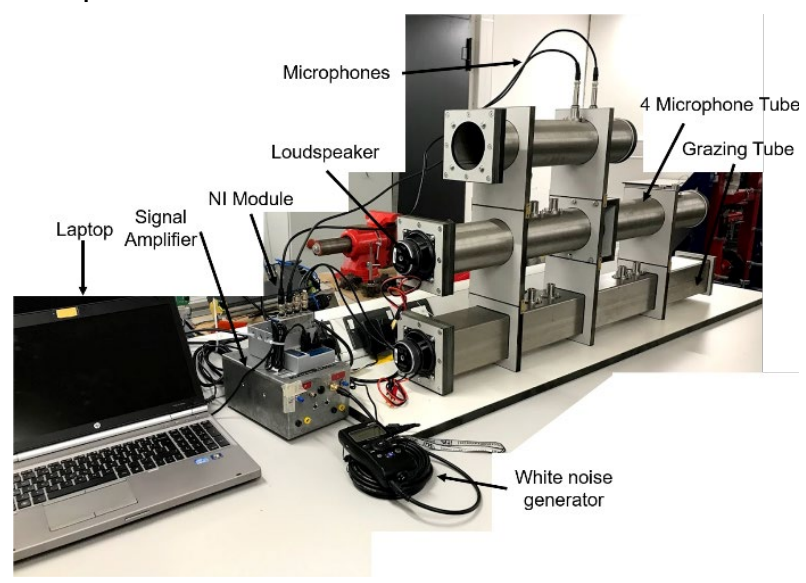

Calculations are then carried out using a complex transfer function to determine the normal incidence absorption and impedance ratios of the acoustic material. (Equation 3-1, Equation 3-2) The usable frequency range depends on the width of the tube and the spacing between the microphones (Figure $3-2$ ) [17]. In this case the diameter of $100 \mathrm{~mm}$ and length $500 \mathrm{~mm}$ giving a working range of $200-2000 \mathrm{~Hz}$.

Figure 3-1 Photograph of experimental rig

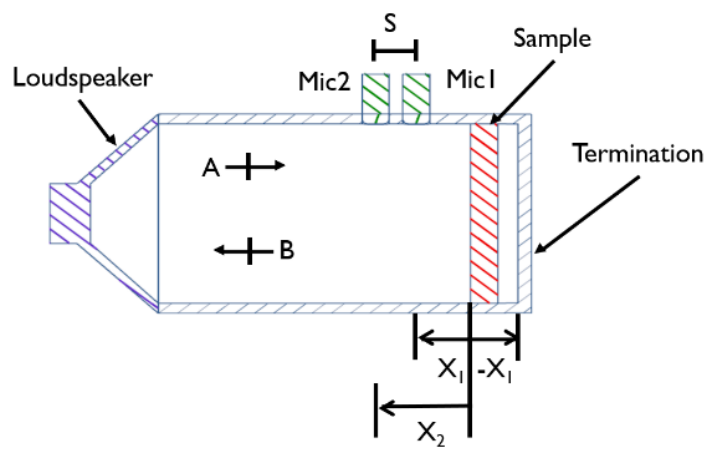

Equation 3-1- Transfer Matrix Two Microphone Impedance Tube

$$
\left[\begin{array}{l}
p \\
v
\end{array}\right]_{x=0}=\left[\begin{array}{ll}
T_{11} & T_{12} \\
T_{21} & T_{22}
\end{array}\right]\left[\begin{array}{l}
p \\
v
\end{array}\right]_{x=d}
$$

Equation 3-2- Sound Transmission Loss

$$
S T L=10 \log _{10}\left(\frac{1}{\tau}\right)
$$

Figure 3-2- Two Microphone Impedance Tube

\section{EXPERIMENTAL ANALYSIS OF LINERS}

\subsection{Methodology}

The experimental analysis determined the reflection and absorption coefficients of each liner by frequency and as a mean value. The data was then compared between the liners and the simulation results in the comparison papers. The liner samples were designed such that each core liner with a diameter of $95 \mathrm{~mm}$ and a rigid backplate of $2.5 \mathrm{~mm}$. An additional variable of three designed top plates of thickness $2.5 \mathrm{~mm}$ and no top plate options were investigated. Each liner was tested with no top plate, the HRTP (Figure 4-1) and a many hole top plate. The labyrinthine used LTP (Figure 4-3) rather than MHTP (Figure 4-2) to follow its specific path route. All components where printed using a Connex 260 Vero White. The experimental analysis of all four liners followed the ISO10534-2 [17] for a two microphone impedance tube. The weights can be found in Table 4-1.

Table 4-1 Acoustic liner and top plate weights

\begin{tabular}{|l|l|l|l|}
\hline LINERS & WEIGHT (G) & TOP PLATES & WEIGHT (G) \\
\hline Helmholtz Resonator & 50 & $\begin{array}{l}\text { Helmholtz } \\
\text { Resonator }\end{array}$ & 20 \\
\hline Hexagonal & 70 & Multi-hole & 21 \\
\hline Labyrinthine & 112 & Labyrinthine & 18 \\
\hline Zig-Zag & 36 & & \\
\cline { 1 - 3 } & & &
\end{tabular}

Vol. 42. Pt. 1. 2020 


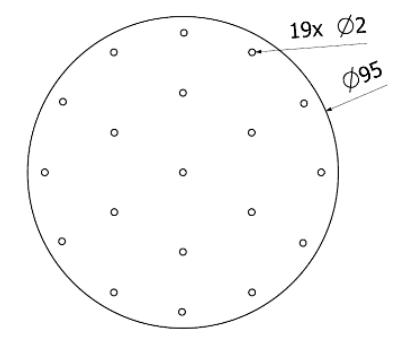

Figure 4-1 Helmholtz resonator top plate

Helmholtz Resonator Top

Plate (HRTP). This perforated top sheet is designed specifically for the Helmholtz resonator.

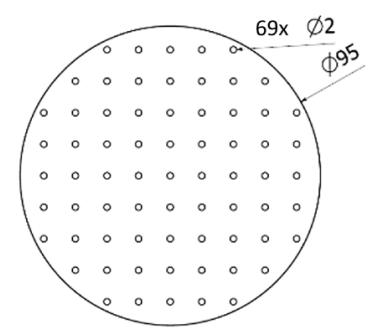

Figure 4-2 Multiple hole top plate

Multiple Hole Top Plate

(MHTP). This perforated top sheet was designed to have the same orifice diameter as the HRTP page but 60 holes.

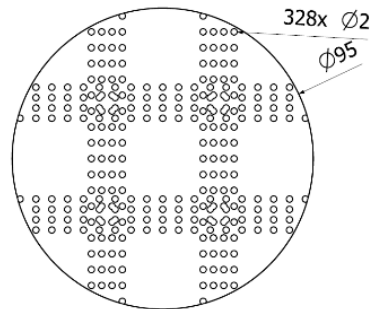

Figure 4-3 Labyrinthine top plate

Labyrinthine Top Plate (LTP). This perforated top sheet was designed to have the same orifice diameter but aligned to the wave path of the acoustic liner with 328 holes.

\subsection{Results}

Figure 4-4 shows absorption coefficients for each liner in each top plate configuration. The non-top plate option is tested for all of the liners for comparison to origin papers, but as the flow orientation is head on rather than grazing the resulting acoustic profile is very different. This impedance test was originally designed for the traditional acoustic liner in sandwich configuration therefore liner designs where tested both with and without top plates. It is planned to further test formations in grazing conditions to capture a full acoustic profile for all potential flow operating conditions. From Figure 4-4 can be seen that for all designs the without a top plate do not display resonant conditions. The impedance effect is material-based absorption peak at a frequency of $580 \mathrm{~Hz}$ with an amplitude of absorption coefficient of 0.25 consistently.
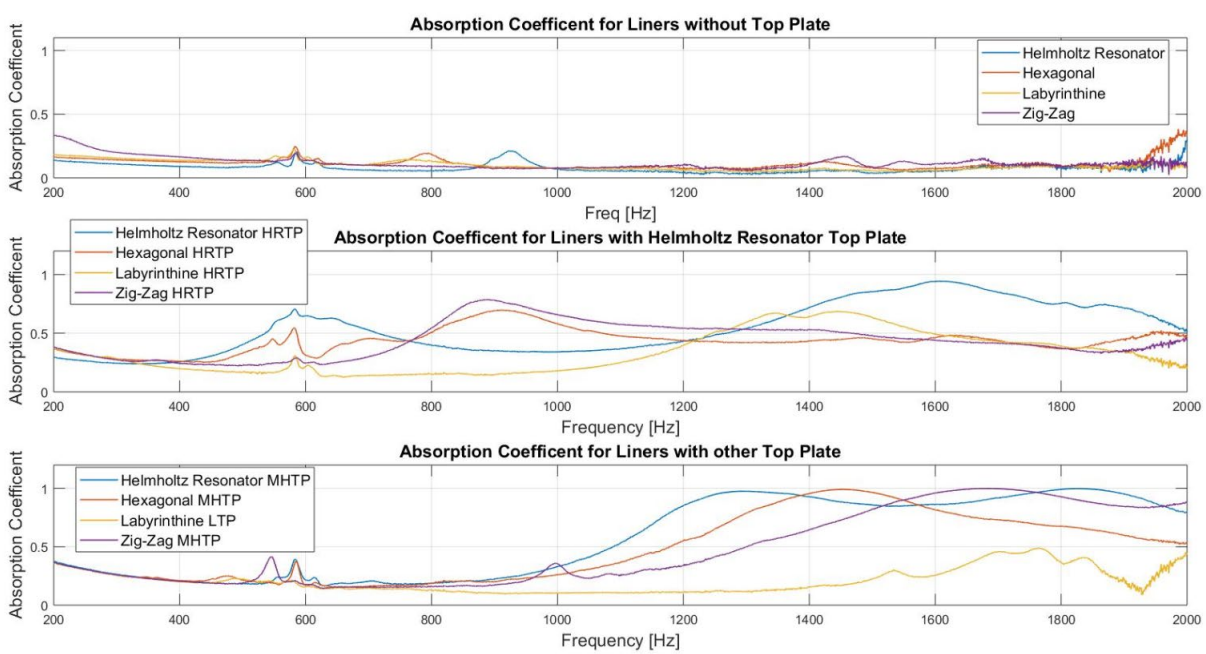

Figure 4-4 Experimental Absorption Coefficient

The peak frequencies are much broader than predicted and with additional multiple peaks. These are anticipated to be due to structural resonances. For consistency, no design factors such as framing or voids were used and unit patterns where tessellated to the edges of the samples (see Figure 4-4). The free walls are excited by the flow through the top plate and around the edge of the tube causing additional dominant peaks. This theory will be tested in future work when design factor such as framing are implemented. Additionally, the core and top-plate are only temporally fixed rather than bonded due to limited printing resources which can lead to flow creep and coupling

Vol. 42. Pt. 1. 2020 
between units. Also,there part sized units caused by the too edge tessellations contribute to absorption characteristics at higher then design resonant frequency. The result of these factors is a non-ideal resonant plot, but they are more consistent with working conditions where samples are cut to fit or degrade over time, thus an understanding of the impact is valuable.

\subsubsection{Mean reflection and absorption coefficients}

Figure 4-6 displays the mean acoustic coefficient values for all sample configurations. For all configurations the introduction of a top-plate increases the absorption coefficient significantly, it is also generally true that increasing the number of holes in the top plate increases the overall absorption, but shifts the resonant frequency to higher values. The shift to higher frequencies as established by the work of Bies and Handen [18] and will be further investigated in later experimental phases.

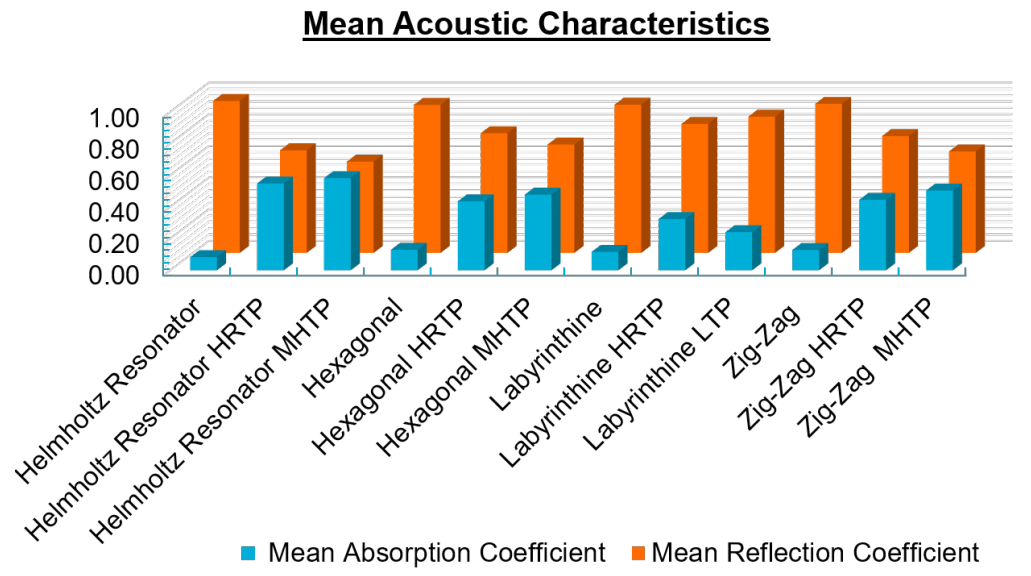

Figure 4-5 Mean reflection and absorption coefficient values

The aim of this research is to propose an SFC acoustic liner that is comparable or better than the baseline HR. The success criteria is an increased absorption coefficient at targeted low frequencies; at a reduced depth and weight. No SFC designs outperformed the traditional HR in absorption coefficient in these flow conditions. The best performing SFC configuration is the zig-zag MHTP at a $5 \mathrm{~mm}$ reduction in depth and $14 \mathrm{~g}$ reduction in weight. This indicates the potential of the SFC within resonant characteristics which when combined with the sub- wavelength mechanism could produce a very effective acoustic liner for a jet engine inlet.

The labyrinthine design performs poorly as a sound absorber for both top plate configurations. It was concluded that due to the high filling ratio of the labyrinthine the material properties dominate in a head on flow. The wave instead of travelling through the path and being attenuated through resonant or sub- wavelength mechanisms is being reflected back by the high volume of material. As a result, the low filing ratio design will be favored in future prototyping stages.

\subsubsection{Resonant Peak}

Figure 4-7 illustrates the comparisons of experimental and simulation (determined from origin journals) resonant peaks. The experimental data points are sized according to absorption coefficient to capture the weight for acoustic significance. The simulation data points are all set to 1 as a coefficient. As well as offering a comparison to the origin paper research this plot was used an experimental validation. The baseline $\mathrm{HR}$ has a design peak of $610 \mathrm{~Hz}$ however, due to printing tolerances this accepted value for the resonance is $580-650 \mathrm{~Hz}$ which the HR HRTP sample met.

Vol. 42. Pt. 1. 2020 
Although there is an additional peak due to structural resonance this does not affect the validity of the experimental method.

Generally, the peak frequencies in the paper do not align with the experimental resonant peaks indicating the grazing flow peak does not determine head on peak value. Conversely the use of MHTP showed improved more aligned peak values for the hexagonal and zig-zag designs demonstrating that design factors such as perforation number could be utilized for increasing liner effectiveness in all flow orientations.

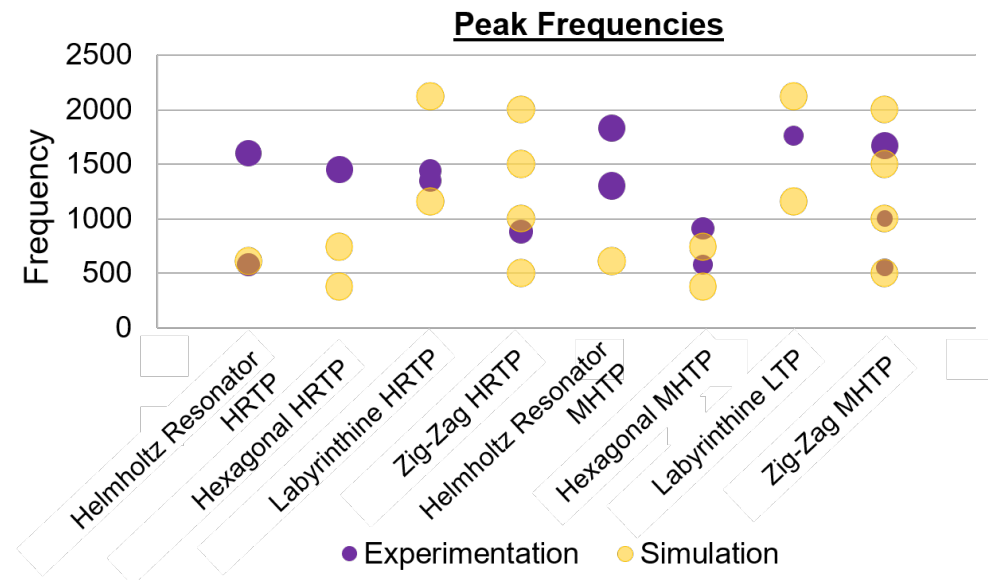

Figure 4-6 Absorption coefficient frequency peak comparison of computational and experimental data

\section{CONCLUSION}

The frequency agreement between the simulation data presented in the papers and this comparison was generally poor demonstrating the large variation in attenuation due to testing or flow conditions. This also highlights the importance of experimental grazing flow testing to understand the full acoustic profile of design and performance in all working flow conditions. Although the models could not be perfectly recreated, as they were simulated in 2D and reproduced in 3D, the absorption peaks were consistently at a higher frequency than predicted and did not reliably show the multiple resonant characteristics being highly influenced by top plate configuration. The experimental performance reinforced the theoretical difficulty of attenuating at low frequencies below $1000 \mathrm{~Hz}$ whilst maintaining restricted liner depth. As a result, an accurate computational simulation is another pivotal progression of this research. The absorption performance of the zig-zag liner showed the promise of SFC's being comparable to the baseline HR at a reduced weight and depth which with further design iterations could lead to an effective liner solution for jet engine inlet now and in the future.

\section{FUTURE WORK}

As stated in the conclusion, the key progression for this work is an accurate computer simulation which will be produced on OpenFOAM software tuned to give comparable results to industrystandard two microphone and four microphone impedance tube tests. The metamaterial liner design will focus on adaptations of existing space filling curves with flow path tuned to a low frequency range specific to jet engine inlets as prescribed by work by Khardi [19]. For example, adding sample frames to the liner samples or varying unit scales. The experimental research will progress onto four microphone impedance tubes and grazing flow tests which are more comparable to jet engine inlet working conditions.

\section{REFERENCES}

Vol. 42. Pt. 1. 2020 
[1] UK Government, "Noise pollution: economic analysis," 2013. [Online]. Available:

https://www.gov.uk/guidance/noise-pollution-economic-analysis. [Accessed: 09-Apr-2018].

[2] N. Pignier, "The impact of traffic noise on economy and environment: a short literature study," Stockholm, 2015.

[3] ACARE, "Strategic Research Agenda Volume 1," Strateg. Res. Agenda, p. 153, 2002.

[4] G. Pagnano, "Clean Sky Contribtution Session:Strategy for effective noise control," in Noise in Europe, 2017.

[5] B. R. Agrawal, "Modeling fan broadband noise from jet engines and rod-airfoil benchmark case for broadband noise prediction," 2015.

[6] G. Huang and P. Frank Pai, Theory and Design of Acoustic Metamaterials. Bellingham: Society of Photo-Optical Instrumentation Engineers Press Press, 2015.

[7] M. . Mokbel, W. . Aref, and I. Kamel, "Analysis of Multi-Dimensional Space-Filling Curves," Geoinformatica, no. October 2003, pp. 179-209, 2003.

[8] D. Wu, N. Zhang, C. M. Mak, and C. Cai, "Noise Attenuation Performance of a Helmholtz Resonator Array Consist of Several Periodic Parts," Sensors MDPI, vol. 5, no. 17, 2017.

[9] S. . Faruq, "An Experimental Investigation on Noise Reduction by Using Modified Helmholtz Resonator," Bangladesh University of Engineering and Technology, 2014.

[10] Y. Wang et al., "A renewable low-frequency acoustic energy harvesting noise barrier for high-speed railways using a Helmholtz resonator and a PVDF film," Appl. Energy, vol. 230, pp. 52-61, 2018.

[11] X. Wang, Y. Zhou, J. Sang, and W. Zhu, "A generalized model for space-coiling resonators," Appl. Acoust., vol. 158, p. 107045, 2020.

[12] B. Xia, H. Dai, and D. Yu, "Symmetry-broken metamaterial for blocking , cloaking , and supertunneling of sound in a subwavelength scale," Appl. Phys. Lett., vol. 251902, no. June, 2016.

[13] C. Liu, B. Xia, and D. Yu, "The spiral-labyrinthine acoustic metamaterial by coiling up space," Phys. Lett. A, vol. 381, no. 36, pp. 3112-3118, 2017.

[14] A. O. Krushynska, F. Bosia, and N. M. Pugno, "Labyrinthine acoustic metamaterials with space-coiling channels for low-frequency sound control," Acta Acust. united with Acust., vol. 104, no. 2, pp. 200-210, 2018.

[15] Y. Cheng, C. Zhou, B. G. Yuan, D. J. Wu, Q. Wei, and X. J. Liu, "Ultra-sparse metasurface for high reflection of low-frequency sound based on artificial Mie resonances," Nat. Mater., vol. 14, no. 10, pp. 1013-1019, 2015.

[16] Y. Xie, B. I. Popa, L. Zigoneanu, and S. A. Cummer, "Measurement of a broadband negative index with space-coiling acoustic metamaterials," Phys. Rev. Lett., vol. 110, no. 17, pp. 1-4, 2013.

[17] International originisation of standards, "ISO 10534-2 Acoustics-Determination of sound absoprtion coefficent and impedance in impedance tubes- Part 2: Transfer-function methord," Geneve, 1998.

[18] D. A. Bies and C. H. Hansen, "Muffeling Devices," in Engineering Noise Control: Theory and Practice, 4th ed., CRC Press, 2009.

[19] S. Khardi, "An Experimental Analysis of Frequency Emission and Noise Diagnosis of Commercial Aircraft on Approach," Jounal Acoust. Emiss., vol. 26, no. 2008, pp. 290-310, 2008. 